\title{
Optimizing Scanning Strategies: Selecting Scanning Bandwidth in Adversarial RF Environments
}

\author{
Andrey Garnaev \\ St. Petersburg State University, Russia \\ Email: garnaev@yahoo.com
}

\author{
Wade Trappe, Chun-Ta Kung \\ WINLAB and the Electrical and Computer Engineering Department \\ Rutgers University, USA \\ Email: trappe@winlab.rutgers.edu, chuntakung@gmail.com
}

\begin{abstract}
In this paper we investigate the problem of designing a spectrum scanning strategy to detect an intelligent Invader who wants to utilize spectrum undetected for his/her unapproved purposes. To deal with this problem we apply game-theoretical tools. We model the situation as a game between a Scanner and an Invader where the Invader faces a dilemma: the more bandwidth the Invader attempts to use leads to a larger payoff if he is not detected, but at the same time also increases the probability of being detected and thus fined. Similarly, the Scanner faces a dilemma: the wider the bandwidth scanned, the higher the probability of detecting the Invader, but at the expense of increasing the cost of building the scanning system. The equilibrium strategies are found explicitly and reveal interesting properties. In particular, we have found a discontinuous dependence of the equilibrium strategies on the network parameters, fine and the type of the Invader's award. This discontinuity on fine means that the network provider has to take into account a human factor since some threshold values of fine could be very sensible for the Invader, while in other situations simply increasing the fine has minimal deterrence impact. Also we show how different reward types for the Invader (e.g. motivated by using different type of application, say, video-streaming or downloading files) can be incorporated into scanning strategy to increase its efficiency 1 .
\end{abstract}

\section{INTRODUCTION}

Over the last few decades, the increasing demand for wireless communications has motivated the exploration for more efficient usage of spectral resources ([11], [15]). In particular, it has been noticed that there are large portions of spectrum that are severely under-utilized [2]. Recently, cognitive radio technologies (CR) have been proposed as a means to intelligently use such spectrum opportunities by sensing the radio environment and exploiting available spectrum holes for secondary usage[6]. In CR systems, secondary users are allowed to "borrow (or lease)" the usage of spectrum from primary users (licensed users), as long as they do not hinder in the proper operation of the primary users' communications. Unfortunately, as we move to make CR technologies commercial, which will allow secondary users to access spectrum owned by primary users, we will face the inevitable risk that adversaries will be tempted to use CR technology for illicit and selfish purposes [14]. If we imagine an unauthorized user

${ }^{1}$ This is the last draft version of the paper. Revised version of the paper accepted by CrownCom 2013 can be found at http: //ieeexplore.ieee.org/xpl/login.jsp?tp=\&arnumber $=6636809 \& u r l=h t t p \% 3 \mathrm{~A} \%$ 2F\%2Fieeexplore.ieee.org\%2Fxpls\%2Fabs_all.jsp\%3Farnumber\%3D6636809
(Invader) attempting to sneak usage of spectrum without obeying proper regulations or leasing the usage of the spectrum, the result will be that both legitimate secondary users and primary users will face unexpected interference, resulting in significant performance degradation across the system.

The challenge of enforcing the proper usage of spectrum requires the notion of a "spectrum policing agent", whose primary job is to ensure the proper usage of spectrum and identify anomalous activities occurring within the spectrum[14]. As a starting point to being able to police the usage of spectrum, we must have the ability to scan spectrum and effectively identify anomalous activities. Towards this objective, there has been several research efforts in signal processing techniques that can be applied to the spectrum scanning problem. For example, in [19], [18], the author's presented methods for detecting a desired signal contained within interference. Similarly, detection of unknown signals in noise without prior knowledge of authorized users was studied in [4], [17]. As another example, in [14], the authors proposed a method to detect anomalous transmission by making use of radio propagation characteristics. In [10] authors investigated what impact on spectrum scanning can have information about the over-arching application that a spectrum thief might try to run.

However, these works tend to not examine the important "interplay" between the two participants inherent in the problem- the Invader, who is smart and will attempt to use the spectrum in a manner to minimize the chance of being detected and fined, while also striving to maximize the benefit he/she receives from illicit usage of this spectrum; and the Scanner, who must be smart and employ a strategy that strategically maximizes the chance of detecting and fining the smart Invader, with minimal cost. This challenge is made more difficult by the complexity of the underlying scanning problem itself: there will be large swaths of bandwidth to scan, and the system costs (e.g. analog-to-digital conversion, and the computation associated with running signal classifiers) associated with scanning very wide bandwidth makes it impossible to scan the full range of spectrum in a single instance. Consequently, it is important to understand the strategic dynamics that exist between the Scanner and the Invader, while also taking into account the underlying costs and benefits that exist for each participant. This paper examines the interactions between the Scanner and Invader by formulating the problem using game theory. We find the optimal scanning strategy by selecting the 
scanning (and, similarly, the invading) bandwidth that should be employed in spectrum scanning.

The organization of this paper is as follows: in Section II we first define the problem by formulating a two-step game in terms of payoff and cost functions: in the first step, each player chooses their bandwidths; while in the second step each player uses the first step results to arrive at equilibrium strategies. We supply saddle point strategies for the game's first step. To gain insight into the problem, for the second step, in Section IV-A we outline a linearized model of detection probability and arrive at the corresponding best strategies for each player in Section IV-B We then explicitly obtain the equilibrium strategies in Sections IV-C and IV-D for cases involving complete and incomplete knowledge of the Invader's reward type. In Section $\mathrm{V}$ numerical illustrations are supplied. Finally, in Sections VI and VII discussions, conclusions and the proofs of the announced results are offered to close the paper.

\section{Formulation of the Scanning Problem as a TWO-STEP GAME}

In this section we set up our problem formulation. Our formulation of the spectrum scanning problem involves two players: the Scanner and the Invader. The Scanner, who is always present in the system, scans a part of the band of frequencies that are to be monitored, in order to prevent illegal usage by a potential Invader of the primary (Scanner) network's ownership of this band. We assume that the amount of bandwidth that needs to be scanned is much larger than is possible using a single scan by the Scanner, and hence the Scanner faces a dilemma: the more bandwidth that is scanned, the higher the probability of detecting the Invader, but at the expense of increasing the cost of the RF scanning system.

We assume that if the Scanner scans a particular frequency band $I_{S}$ and the Invader uses the band $I_{I}$ then the invasion will be detected with certainty if $I_{S} \cap I_{I} \neq \emptyset$, and it will not be detected otherwise. Without loss of generality we can assume that the size of the protected frequency band is normalized to 1 . The Invader wants to use spectrum undetected for some illicit purpose. We consider two scenarios: (a) The reward for the Invader is related to the width of the frequency band he uses if he is undetected. If he is detected he will be fined. So, the Invader faces a dilemma: the more bandwidth he tries to use yields a larger payoff if he is not detected but also it increases the probability of being detected and thus to be fined, (b) The award for the Invader is unknown to the Scanner: he only knows whether it is related to the width of the frequency band the Invader uses, or not.

We formulate this problem as a two step game in the following two subsections.

\section{A. Formulation of the Problem in the First Step of the Game}

In the first step of the game the Scanner selects the band $B_{S}=\left[t_{S}, t_{S}+x\right] \subseteq[0,1]$ with a fixed upper bound of frequency width $x$ to scan i.e. $t_{S} \leq 1-x$. The Invader selects the band $B_{I}=\left[t_{I}, t_{I}+y\right] \subseteq[0,1]$ with a fixed upper bound frequency width $y$ to intrude, i.e. $t_{I} \leq 1-y$. So, $B_{S}$ and $B_{I}$ are pure strategies for the Scanner and the Invader. The Scanner's payoff $v\left(B_{S}, B_{I}\right)$ is 1 if the Invader is detected (i.e. $\left.\left[t_{S}, t_{S}+x\right] \cap\left[t_{I}, t_{I}+y\right] \neq \emptyset\right)$ and his payoff is zero otherwise. The goal of the Scanner is to maximize his payoff meanwhile the Invader wants to minimize it. So, the Scanner and the Invader play a zero-sum game. The saddle point (equilibrium) of the game is a couple of strategies $\left(B_{S_{*}}, B_{I *}\right)$ such that for each strategies $\left(B_{S}, B_{I}\right)$ the following inequalities hold [16]:

$$
v\left(B_{S}, B_{I *}\right) \leq v:=v\left(B_{S *}, B_{I *}\right) \leq v\left(B_{S *}, B_{I}\right),
$$

where $v$ is the value of the game. It is clear that the game does not have a saddle point in the pure strategy if $x+y \leq 1$. To find the saddle point we have to extend the game by mixed strategies, where we assign a probability distribution over pure strategies. Then instead of the payoff $v$ we have its expected value. The game has a saddle point in mixed strategies, and let $P(x, y)$ be the value of the game. Then $P(x, y)$ is the maximal detection probability of the Invader under worst conditions.

\section{B. Formulation of the Problem in the Second Step of the Game}

In the second step of the game the rivals knowing their equilibrium strategies from the first step as well as detection probability $P(x, y)$, want to find the equilibrium frequency widths $x$ and $y$. We here consider two sub-scenarios: (a) the Invader's type is known: namely, it is known how the reward for the Invader is related to the width of the frequency band he uses if he is undetected, (b) the Invader's type is unknown: instead, there is only a chance that the Invader reward is related to the width in use, else it is not related. Different type of rewards can be motivated by using different type of application (say, file-download or streaming video [10]).

1) Invader reward is related to the bandwidth used: A strategy for the Scanner is to scan a width of frequency of size $x \in[a, b]$, and a strategy for the Invader is to employ a width of frequency of size $y \in[a, c]$, where $c<b<1 / 2$. So, we assume that the Invader's technical characteristics is not better than the Scanner's ones.

If the Scanner and the Invader use the strategies $x$ and $y$, then the payoff to the Invader is the expected award (which is a function $U(y)$ of bandwidth $y$ illegally used by the Invader) minus intrusion expenses (which is a function $C_{I}(y)$ of bandwidth $y$ ) and expected fine $F$ to pay, i.e.

$$
v_{I}(x, y)=(1-P(x, y)) U(y)-F P(x, y)-C_{I}(y) .
$$

The Scanner wants to detect intrusion taking into account scanning expenses and damaged caused by the illegal use of the bandwidth. For detection he is rewarded by fined $F$ imposed on the Invader. Thus, the payoff to the Scanner is difference between the expected reward for detection, and damaged from intrusion into the bandwidth (which is a function $V(y)$ of bandwidth $y$ illegally used by the Invader) with the scanning expenses (which is a function $C_{S}(x)$ of scanned bandwidth $x$ ),

$$
v_{S}(x, y)=F P(x, y)-V(y)(1-P(x, y))-C_{S}(x) .
$$


Note that introducing transmission cost is common for CDMA [22] and ALOHA networks ([23], [24]). We assume that the Scanner and the Invader know fine $F$, cost functions $C_{I}$ and $C_{S}$, the Scanner and Invader's utilities $V$ and $U$ as well as low and upper bandwidth bounds $a, b$ and $c$. We look for a Nash equilibrium, i.e. for a couple of strategies $\left(x_{*}, y_{*}\right)$ such that for any couple of strategies $(x, y)$ the following inequalities hold [16]:

$$
v_{S}\left(x, y_{*}\right) \leq v_{S}\left(x_{*}, y_{*}\right), \quad v_{I}\left(x_{*}, y\right) \leq v_{I}\left(x_{*}, y_{*}\right) .
$$

2) Unknown Whether the Invader's Award is Related to the Width of Band in Use: In this section we assume that the Invader can be of two types: (a) with probability $q$ he can be the same as in the previous section, and so $y$ is his strategy and payoffs are given by (1), (b) with probability $1-q$ for him it is just important to work in the network without being detected. Then, of course, he will employ the minimal bandwidth allowed, so his strategy is $y=a$. The payoff to the Scanner is the expected payoff taking into account the type of Invader:

$$
v_{S}^{E}(x, y)=q v_{S}(x, y)+(1-q) v_{S}(x, a)
$$

with $v_{S}(x, y)$ given by (2). Here we also look for Nash equilibrium. We assume that the Scanner and the Invader know (as in the case with complete information) the parameters $F$, $C_{I}, C_{S}, V, U, a, b, c$ as well as the probability $q$.

\section{EQUILIBRIUM STRATEGIES FOR THE FIRST STEP}

In the following theorem we gives the equilibrium strategies for the first step, so for fixed upper bound width of the rivals.

Theorem 1: In the first step with fixed width to scan $x$ and to invade $y$, the rivals employ uniform tiling behavior. Namely,

(a) Let $1-(x+y) M \leq y$ with

$$
M=\lfloor 1 /(x+y)\rfloor
$$

where $\lfloor\xi\rfloor$ is the greatest integer less or equal to $\xi$. Then the Scanner and the Invader will, with equal probability $1 / M$, employ a band of the set $A_{-S}$ and $A_{-I}$ correspondingly.

(b) Let $1-(x+y) M>y$. Then the Scanner and the Invader will, with equal probability $1 /(M+1)$, employ a band of the set $A_{+S}$ and $A_{+I}$ correspondingly, where

$$
\begin{aligned}
& A_{-S}=\{[k(x+y)-x, k(x+y)], k=1, \ldots, M\}, \\
& A_{-I}=\{[k(x+y)-y-\epsilon(M+1-k), k(x+y)-\epsilon(M-k)] \text {, } \\
& k=1, \ldots, M\}, \quad 0<\epsilon<x / M, \\
& A_{+S}=A_{-S} \cup[1-x, 1] \text {, } \\
& A_{+I}=\{[(k-1)(x+y+\epsilon),(k-1)(x+y+\epsilon)+y] \text {, } \\
& k=1, \ldots, M\} \cup[1-y, 1], \quad 0<\epsilon<\frac{1-y-M(x+y)}{M-1} .
\end{aligned}
$$

The value of the game (detection probability) $P(x, y)$ is given as follows:

$$
P(x, y)= \begin{cases}1 / M, & 1-(x+y) M \leq y \\ 1 /(M+1), & 1-(x+y) M>y\end{cases}
$$

\section{THE EQUILIBRIUM STRATEGY FOR THE SECOND STEP}

In this section, which is split into four subsections, we find the equilibrium strategy for the second step explicitly. First in Subsection IV-A we linearize our model to get an explicit solution, then in Subsection IV-B the best response strategies are given for known Invader type, and they are employed in Subsections IV-C and IV-D to construct equilibrium strategies for known and unknown Invader types correspondingly.

\section{A. Linearized model for the second step}

In order to get an insight into the problem, we consider a situation where the detection's probability $P(x, y)$ for $x, y \in$ $[a, b]$ is approximated by a linear function as follows:

$$
P(x, y)=x+y \text {. }
$$

We assume that the scanning and intrusion cost as well as the Invader's and Scanner's utilities are linear in the bandwidth involved, i.e. $C_{S}(x)=C_{S} x, C_{I}(y)=C_{I} y, U(y)=U y$, $V(y)=V y$ where $C_{S}, C_{I}, U, V>0$. Then the payoffs to the Invader and the Scanner, if they use strategies $x \in[a, b]$ and $y \in[a, c]$ respectively, become:

$$
\begin{aligned}
& v_{I}(x, y)=U(1-x-y) y-F(x+y)-C_{I} y, \\
& \text { for known Invader's type: } \\
& v_{S}(x, y)=F(x+y)-V y(1-x-y)-C_{S} x, \\
& \text { for unknown Invader's type: } \\
& v_{S}^{E}(x, y)=q(F(x+y)-V y(1-x-y)) \\
& +(1-q)(F(x+a)-V y(1-x-a))-C_{S} x .
\end{aligned}
$$

Note that linearized payoffs have found extensive usage for a wide array of problems in wireless networks [9], [23], [25], [26], [27]. Of course, such approach simplifies the original problem and only gives an approximated solution. Meanwhile it can also be very useful: sometimes it allows one to obtain solution explicitly, and allows one to look inside of the structure of the solution as well as the correlation between parameters of the system.

\section{B. Best response strategies for the Invader's reward related to bandwidth used}

In this section we give best response strategies for the Scanner and the Invader, i.e. such strategies that $\operatorname{BR}_{S}(y)=$ $\arg \max _{x} v_{S}(x, y)$ and $\mathrm{BR}_{I}(x)=\arg \max _{y} v_{I}(x, y)$.

Theorem 2: In the second step of the game the Scanner and the Invader have the best response strategies $\operatorname{BR}_{S}(y)$ and $\mathrm{BR}_{I}(x)$ are given as follows:

$$
\begin{gathered}
\operatorname{BR}_{S}(y)= \begin{cases}a, & y<\left(C_{S}-F\right) / V, \\
\text { any from }[a, b], & y=\left(C_{S}-F\right) / V, \\
b, & y>\left(C_{S}-F\right) / V,\end{cases} \\
\operatorname{BR}_{I}(x)= \begin{cases}a, & T-2 a \leq x, \\
L(x), & T-2 c<x<T-2 a, \\
c, & x \leq T-2 c\end{cases}
\end{gathered}
$$

with

$$
L(x)=(T-x) / 2, \quad T=\left(U-F-C_{I}\right) / U .
$$




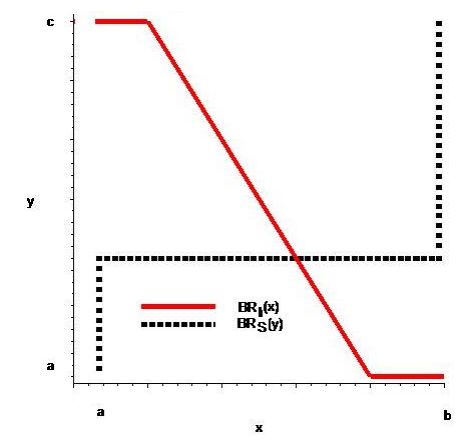

Fig. 1. The Nash equilibrium as an intersection of the best response curves

\section{Equilibrium: the Invader's Award is Related to the Width of Band in Use}

The equilibrium in the second step of the problem exists since the payoff to the Scanner is linear on $x$ and the payoff to the Invader is concave on $y$. The equilibrium can be found by (3) as a couple of strategies $(x, y)$ which are the best response to each other, i.e. $x=\mathrm{BR}_{S}(y)$ and $y=\mathrm{BR}_{I}(x)$, i.e. the intersection of the best response curves (Figure (1). Such intersection always exists and is unique as shown in the following theorem.

Theorem 3: Let the Invader's award be related to the width of band in use. This game has unique Nash equilibrium in the second step, and it is given by Table I with $R=\left(C_{S}-F\right) / V$.

\section{Equilibrium: Unknown whether the Invader's reward is related to bandwidth used}

For marginal probabilities of the problem with unknown Invader's type (where indeed there is complete confidence in the Invader's type) we already have the solution. Namely, if $q=1$ then the Invaders' reward depends on width in use. So, the equilibrium is unique and given by Theorem 3 . If $q=0$ then the Invaders' reward does not depend on width in use.

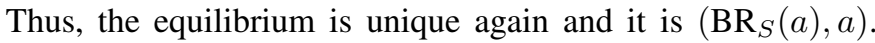
For the inside probabilities the equilibrium is given by the following theorem

Theorem 4: Let it be unknown whether the Invader's award is related to the bandwidth used. This game has unique Nash equilibrium in the second step, and it is given by Table $\mathrm{T}$ with $R=\left(C_{S}-F-(1-q) V a\right) /(q V)$ and $L^{-1}(R)=T-2\left(C_{S}-\right.$ $F-(1-q) V a) /(q V)$.

\section{NUMERICAL ILLUSTRATIONS}

As a numerical illustration of the scenario when the Invader's reward depends on the bandwidth in use we consider $U=V=1, a=0.01, b=0.3, C_{S}=0.4, C_{I}=0.1$. Figures 2 and 3 demonstrate the equilibrium strategies as a function of the fine $F$ for the Invader's characteristics $c=0.2,0.3$. Increasing fine makes the Scanner and the Invader employ larger and smaller bands correspondingly. The Scanner would alter his strategy by a sudden jump at a threshold value $(F=0.1(0.2)$ for $c=0.2(0.3))$. In spite of the fact that the Invader would vary his strategy continuously, his payoff experiences a sudden drop. This also leads to an increase in

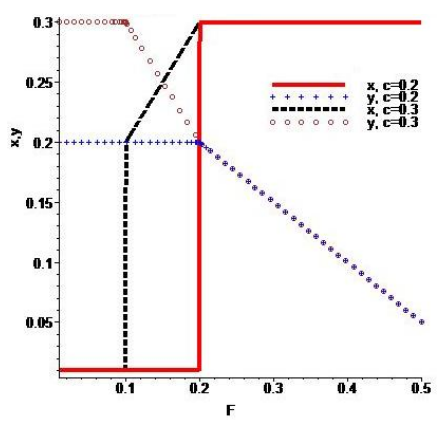

Fig. 2. Equilibrium strategy $x$ and $y$ when the Invader's reward depends on the bandwidth in use

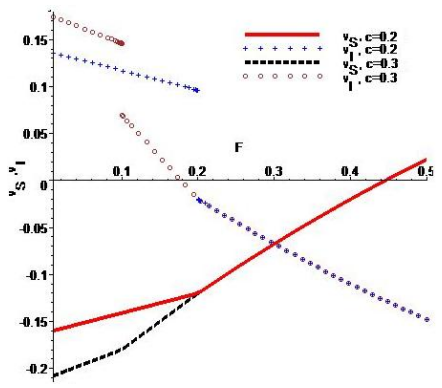

Fig. 3. The equilibrium payoffs $v_{S}$ and $v_{I}$ when the Invader's reward depends on the bandwidth in use

detection probability by a jump. Since the Scanner already gets the upper band, while the Invader still does not get to the lower band, further increasing of the fine leads to continuous decreasing of the detection probability due to the smaller bandwidth employed by the Invader (Figure 4 .

Figure 5 and 6 demonstrate the equilibrium strategies as a function of probability $q$ (i.e. uncertainty about the Invader's reward type) and fine $F=0.2$. The result essentially depends on the Invader's characteristics. For $c=0.2$ the equilibrium strategies do not depend on the probability $q$. For $c=0.3$ they are constant for $q<0.68$, while increasing of the Scanner strategy by a jump at switching point $q=0.68$ drops also the Invader's payoff by a jump. This "jumping nature" for the Invader's payoff means that when assigning a fine one has to take into account the fact that sometimes a simple increase in

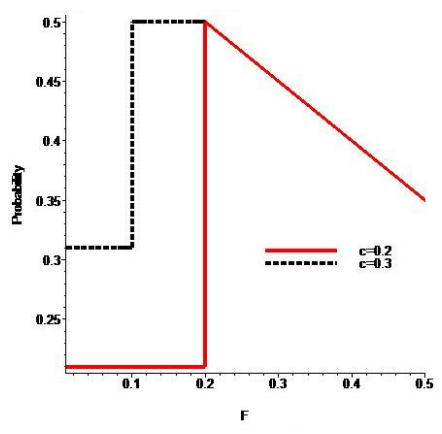

Fig. 4. Probability of detection when the Invader's reward depends on the bandwidth in use 


\begin{tabular}{|c|c|c|c|c|c|}
\hline \hline Case & Condition & Condition & $x$ & $y$ & Detection probability \\
\hline \hline$i_{1}$ & $R<a$ & $L(b)<a$ & $b$ & $a$ & $a+b$ \\
\hline$i_{2}$ & $R<a$ & $a \leq L(b) \leq c$ & $b$ & $L(b)$ & $b+L(b)$ \\
\hline$i_{3}$ & $R<a$ & $c<L(b)$ & $b$ & $c$ & $c+b$ \\
\hline$i_{4}$ & $R>c$ & $L(a)<a$ & $a$ & $a$ & $2 a$ \\
\hline$i_{5}$ & $R>c$ & $a \leq L(a) \leq c$ & $a$ & $L(a)$ & $a+L(a)$ \\
\hline$i_{6}$ & $R>c$ & $c<L(a)$ & $a$ & $c$ & $a+c$ \\
\hline$i_{7}$ & $a \leq R \leq c$ & $L(b) \leq R \leq L(a)$ & $L^{-1}(R)$ & $R$ & $L^{-1}(R)+R$ \\
\hline$i_{8}$ & $a \leq R \leq c$ & $L(a) \leq a$ & $a$ & $a$ & $2 a$ \\
\hline$i_{9}$ & $a \leq R \leq c$ & $a<L(a)<R$ & $a$ & $L(a)$ & $a+L(a)$ \\
\hline$i_{10}$ & $a \leq R \leq c$ & $c<L(b)$ & $b$ & $c$ & $c+b$ \\
\hline$i_{11}$ & $a \leq R \leq c$ & $R<L(b)<c$ & $b$ & $L(b)$ & $b+L(b)$ \\
\hline \hline
\end{tabular}

The Nash equilibrium $(x, y)$ with $L^{-1}(R)=L^{-1}\left(\left(C_{S}-F\right) / V\right)=T-2\left(C_{S}-F\right) / V$.

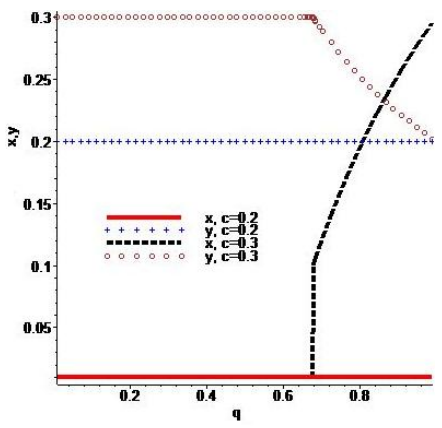

Fig. 5. Equilibrium strategy $x$ and $y$ with uncertainty about the Invader's

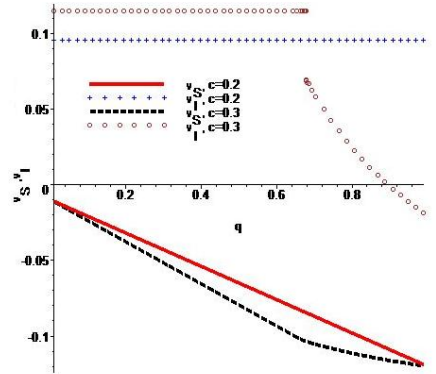

Fig. 6. The equilibrium payoffs $v_{S}$ and $v_{I}$ with uncertainty about the Invader's award

fine might not change the Invader's behavior, but that once a threshold value is hit, his behavior will change dramatically.

\section{DISCUSSION}

In this paper we suggest a simple model of finding the optimal bandwidth to scan for detection of an Invader and found the solution of this model explicitly. We have shown that the optimal width essentially depends on the scenario and under some conditions a small varying of network parameters and fine could lead to jump changes in the optimal strategies, as well as in the payoffs of the rivals. This mixture between continuous and discontinuous behavior of the Invader under the influence of fine implies that the network provider has to carefully make a value judgement: some threshold values of fine could have a huge impact on the Invader, while in the other

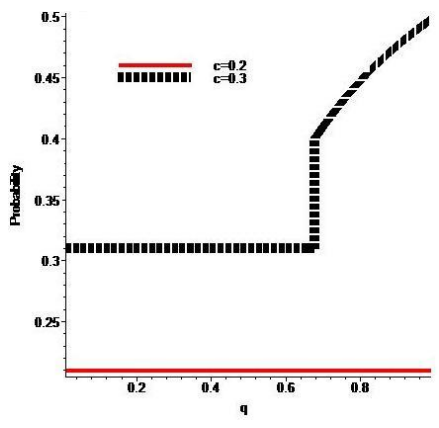

Fig. 7. Probability of detection with uncertainty about the Invader's award

situations a small increase will have a minimal impact on the strategies used. A goal for our future investigation, of course, is to investigate the non-linearized detection probability. Also, since our investigation showed that the optimal scanning essentially depends on the Invader's characteristics, we intend to extend our model to the case of incomplete information on the characteristics as well as to multi-scanner [5], [7] or multi-invader systems [8].

\section{REFERENCES}

[1] Adlakha, S., Johari, R., and Goldsmith, A., Competition in wireless systems via bayesian inference games. CoRR abs/0709.0516, 2007.

[2] Akyildiz, I.F., Lee, W.Y., Vuran, M.C., and Mohanty, S., Next generation /dynamic spectrum access/ cognitive radio wireless networks: a survey. Computer Networks,50, 2127-2159, 2006.

[3] Brown, T.X., Sethi, A., Potential cognitive radio denial-of-service vulnerabilities and protection countermeasures: A multi-dimensional analysis and assessment. Mobile Networks and Applications, 13, 516532, 2008.

[4] Digham, F.F., Alouini, M.S., and Simon, M.K., On the energy detection of unknown signals over fading channels. IEEE Transactions on Communications, 55, 21-24, 2007.

[5] Comaniciu, C., Mandayam, N.B., and Poor, H.V., Wireless Networks Multiuser Detection in Cross-Layer Design, Springer, 2005

[6] Fette, B.A., Cognitive radio technology, Academic Press, 2009

[7] Garnaev, A., On a Ruckle problem in discrete games of ambush. Naval Research Logistics, 44, 353-364, 1997.

[8] Altman E., Avrachenkov K., Garnaev A., Jamming in Wireless Networks: the Case of Several Jammers. GameNets 2009, pp.585-592.

[9] Altman, E., Avrachenkov, K., and Garnaev, A., Jamming in Wireless Networks Under Uncertainty. Mobile Networks and Applications, 16, 246-254, 2011. 
[10] Garnaev, A., Trappe, W., and Kung, C.-T., Dependence of Optimal Monitoring Strategy on the Application to be Protected. IEEE GlobeCom 2012, pp. 1072-1077.

[11] Haykin, S., Cognitive radio: brain-empowered wireless communications. IEEE Journal on Selected Areas in Communications, 23, 201-220, 2005.

[12] Heikkinen, T., A Minmax Game of Power Control in a Wireless Network under Incomplete Information. DIMACS Tech. Report 99-43, 1999.

[13] Jean, S., and Jabbari, B., Bayesian Game-theoretic Modeling of Transmit Power Determination in a Self-organizing CDMA Wireless Network. IEEE VTC 2004.

[14] Liu, S., Chen, Y., Trappe, W., and Greenstein, L.J., Aldo: An anomaly detection framework for dynamic spectrum access networks. INFOCOM 2009.

[15] Mitola, J., Cognitive radio for flexible mobile multimedia communications. IEEE International Workshop on Mobile Multimedia Communications 1999, pp.3-10.

[16] Owen, G., Game Theory. W.B.Sanders, Philadelphia, 1982.

[17] Urkowitz, H., Energy detection of unknown deterministic signals. Proceedings of the IEEE, 55, 523-531, 1967.

[18] Van Trees, H.L., Detection, Estimation, and Modulation Theory, RadarSonar Signal Processing and Gaussian Signals in Noise. WileyInterscience, 2004.

[19] Verdu, S., Multiuser detection. Cambridge Univ Press, 1998.

[20] Wang, B., Wu, Y., and Liu, K.J., Game theory for cognitive radio networks: An overview. Computer Networks, 54, 2537-2561, 2010.

[21] Xing, Y. and Mathur, C.N. and Haleem, MA and Chandramouli, R. and Subbalakshmi, KP, Dynamic spectrum access with QoS and interference temperature constraints. IEEE Transactions on Mobile Computing, 6 , 423-433, 2007.

[22] Zhu, Q., Saad, W., Han. Z., Poor, H.V., and Basar, T., Eavesdropping and Jamming in Next-Generation Wireless Networks: A Game-Theoretic Approach. MILCOM 2011.

[23] Sagduyu,Y.E., and Ephremides, A., A Game-Theoretic Analysis of Denial of Service Attacks in Wireless Random Access. Journal of Wireless Networks, 15, 651-666, 2009.

[24] Garnaev A., Hayel Y., Altman E., and Avrachenkov K., Jamming Game in a Dynamic Slotted ALOHA Network. GameNets 2011, pp. 429-443.

[25] Kim, S.L., Rosberg, Z., and Zander, J., Combined power control and transmission selection in cellular networks. IEEE VTC 1999.

[26] Koo, I., Ahn, J., Lee, H.A., and Kim, K.,Analysis of Erlang capacity for the multimedia DS-CDMA systems. IEICE Trans. Fundamentals, E82A(5), pp.849-855, 1999.

[27] Garnaev A., Trappe W., The Eavesdropping and Jamming Dilemma in Multi-Channel Communications. IEEE ICC 2013.

\section{APPENDIX}

\section{A. Proof of Theorem 1}

Suppose that the Invader uses a band $B_{I}$ with width $y$ and the Scanner with equal probability employ a band from the set $A_{-S}\left(A_{+S}\right)$ for $1-(x+y) M \leq y$ (for $1-(x+y) M>y$ ). The intervals composing $A_{-S}$ and $A_{+S}$ are separated from each other by at most $y$. So, at least one band from $A_{-S}$ for $1-(x+y) M \leq y$ and from $A_{+S}$ for $1-(x+y) M>y$ intersects with $B_{I}$. So, detection probability is greater or equal to $1 / M$ for $1-(x+y) M \leq y$ and it is is greater or equal to $1 /(M+1)$ for $1-(x+y) M>y$.

Suppose that the Scanner uses a band $B_{S}$ with width $x$ and the Invader with equal probability employ a band from the set $A_{-I}\left(A_{+I}\right)$ for $1-(x+y) M \leq y$ (for $1-(x+y) M>y$ ). The intervals composing $A_{-I}$ and $A_{+I}$ are separated from each other by more that $x$. So, at most one band from $A_{-I}$ for $1-(x+y) M \leq y$ and from $A_{+I}$ for $1-(x+y) M>y$ intersects with $B_{S}$. So, detection probability is less or equal to $1 / M$ for $1-(x+y) M \leq y$ and it is is less or equal to $1 /(M+1)$ for $1-(x+y) M>y$ and the result follows.

\section{B. Proof of Theorem 2}

Note that $v_{S}(x, y)=x\left(F+V y-C_{S}\right)+y(F-V+V y)$. So, for a fixed $y$ the payoff $v_{S}(x, y)$ is linear on $x$. Thus, $\mathrm{BR}_{S}(y)=\arg \max _{x} v_{S}(x, y)$ is defined by sign of $F+V y-$ $C_{S}$ as it is given by (9).

Note that $v_{I}(x, y)=\left(U(1-x)-F-C_{I}\right) y^{2}-U y^{2}-x F$. So, for a fixed $x$ the payoff $v_{I}(x, y)$ is a concave quadratic polynomial on $y$ getting its absolute maximum at $y=(U)(-$ $\left.x)-F-C_{I}\right) /(2 U)$. Thus, the maximum of $v_{I}(x, y)$ within $[a, c]$ is reached either on its bounds $a$ and $c$ or at $y=(U(1-$ $\left.x)-F-C_{I}\right) /(2 U)$ if it belongs to $[a, c]$ as it is given by 10 .

\section{Proof of Theorem 3}

First note that $(x, y)$ is a Nash equilibrium if and only if it is a solution of equations $x=\mathrm{BR}_{S}(y)$ and $y=\mathrm{BR}_{I}(x)$ with $\mathrm{BR}_{S}(y)$ and $\mathrm{BR}_{I}(x)$ given by Theorem 2 .

By (11) we have that (10) is equivalent to

$$
\mathrm{BR}_{I}(x)= \begin{cases}a, & L(x) \leq a, \\ L(x), & a<L(x)<c, \\ c, & c \leq L(x) .\end{cases}
$$

Let $a>\left(C_{S}-F\right) / V$. By (9) $\mathrm{BR}_{S}(y) \equiv b$. This, jointly with (12), implies $\left(i_{1}\right)-\left(i_{3}\right)$.

Let $\left(C_{S}-F\right) / V>c$. By (9) $\mathrm{BR}_{S}(y) \equiv a$. Then 12 implies $\left(i_{4}\right)-\left(i_{6}\right)$.

Let $a \leq\left(C_{S}-F\right) / V \leq c$. First note $L(x)$ is linear decreasing function from $L(a)$ for $x=a$ to $L(b)$ for $x=b$.

(a) Let $L(b) \leq\left(C_{S}-F\right) / V \leq L(a)$. Then the equation $L(x)=\left(C_{S}-F\right) / V$ has the unique root within $[a, b]$. Thus, 9$]$ and $(12)$ yield $\left(i_{7}\right)$.

(b) Let $L(a) \leq\left(C_{S}-F\right) / V$. Then, $L(x)<\left(C_{S}-F\right) / V$ for $x \in(a, b]$. So, by 110$), \mathrm{BR}_{I}(x)<c$ for $x \in[a, b]$. Besides, by the assumption, the equation $L(x)=$ $\left(C_{S}-F\right) / V$ does not has root in $[a, b]$. Thus, by 9 $\mathrm{BR}_{S}(y) \equiv a$. So, 12 implies $\left(i_{8}\right)$ and $\left(i_{9}\right)$.

(c) Let $\left(C_{S}-F\right) / V<L(b)$. Then $L(x)>\left(C_{S}-F\right) / V$ for $x \in[a, b)$. Thus, by $[10], \mathrm{BR}_{I}(x)>a$ for $x \in[a, b]$. Besides, by the assumption, the equation $L(x)=\left(C_{S}-F\right) / V$ does not has root in $[a, b]$. So, by (9), $\mathrm{BR}_{S}(y)=b$, and, 12 implies $\left(i_{10}\right)$ and $\left(i_{11}\right)$.

\section{Proof of Theorem 4}

Note that $v_{S}^{E}(x, y)=\left(F-C_{S}+(1-q) V a+V q y\right) x+q y(F-$ $V+V y)+(1-q) a(F-V+V a)$. So, for a fixed $y$ the payoff $v_{S}^{E}(x, y)$ is linear on $x$. Thus, $\operatorname{BR}_{S}^{E}(y)=\arg \max _{x} v_{S}^{E}(x, y)$ is given as follows

$$
\operatorname{BR}_{S}^{E}(y)= \begin{cases}a, & y<R, \\ \text { any from }[a, b], & y=R, \\ b, & y>R\end{cases}
$$

with $R=\left(C_{S}-F-(1-q) V a\right) /(q V)$. This, jointly with Theorem 2 and the proof of Theorem 3 implies the result. 